\title{
Globe
}

Revue internationale d'études québécoises

\section{Gérard Bouchard et Charles Taylor : Fonder l'avenir. Le temps de la conciliation. Rapport de la Commission de consultation sur les pratiques d'accommodement reliées aux différences culturelles, Québec, 2008}

\section{Georges Leroux}

Volume 12, numéro 1, 2009

URI : https://id.erudit.org/iderudit/1000775ar

DOI : https://doi.org/10.7202/1000775ar

Aller au sommaire du numéro

Éditeur(s)

Globe, Revue internationale d'études québécoises

ISSN

1481-5869 (imprimé)

1923-8231 (numérique)

Découvrir la revue

Citer ce compte rendu

Leroux, G. (2009). Compte rendu de [Gérard Bouchard et Charles Taylor : Fonder l'avenir. Le temps de la conciliation. Rapport de la Commission de consultation sur les pratiques d'accommodement reliées aux différences culturelles, Québec, 2008]. Globe, 12(1), 167-176.

https://doi.org/10.7202/1000775ar d'utilisation que vous pouvez consulter en ligne.

https://apropos.erudit.org/fr/usagers/politique-dutilisation/ 


\section{RECENSIONS}

Frédéric Rondeau [éd.]

+ + + + + + + + + + + + + + + + + + + + + + +

Relire le rapport de la Commission Bouchard-Taylor

Gérard Bouchard et Charles Taylor

Fonder l'avenir. Le temps de la conciliation.

Rapport de la Commission de consultation sur les pratiques

d'accommodement reliées aux différences culturelles

Québec, 2008.

Relire, une année après sa parution, le rapport de la Commission Bouchard-Taylor, c'est d'abord retrouver le climat social et politique dans lequel le Québec s'est trouvé plongé après que le gouvernement eut donné mandat à la Commission de réfléchir sur les normes de la vie collective. $\mathrm{Ce}$ climat fut celui d'une longue conversation publique, ponctuée par des forums citoyens, parfois houleux, où chacun pouvait prendre la parole, mais surtout par un débat très médiatisé où s'affrontaient plusieurs conceptions de la laïcité et de l'intégration. Le mandat de la Commission était clair et son application limitée : une série d'affaires, pour la plupart devenues publiques entre 1985 et 2007 , avaient permis de mesurer la multiplication de points de friction, suite à des demandes d'accommodement relatives soit à des règlements, soit à des lois en matière de culture et de religion. Quand on revoit la chronologie de ces affaires, telle que les commissaires la reconstituent dans la deuxième partie de leur rapport (p. 43-60), on peut s'accorder avec le diagnostic fondamental de la Commission : aucun de ces dossiers ne constituait un facteur de déstabilisation des normes collectives régissant la vie sociale au Québec, et plusieurs cas révèlent à l'analyse une inflation résultant de leur insertion dans un ensemble très médiatisé. Cela dit, après avoir passé en revue chacun de ces cas et récapitulé les pratiques d'harmonisation effectives 
dans les institutions publiques, les commissaires ont proposé, dans la troisième partie du rapport, un vaste cadre de référence (p. 101-154) qu'on peut considérer comme la contribution la plus importante de la Commission à la réflexion collective. C'est ce texte auquel je voudrais revenir.

Formé de trois chapitres inspirés par une philosophie sociale et politique pluraliste, ce cadre présente les principes fondamentaux de la démocratie libérale au Québec : d'une part, la prépondérance de la Charte québécoise des droits de la personne et des droits de la jeunesse et de la Charte de la langue française, et d'autre part les principes de l'intégration et de la laïcité. Parmi les questions soulevées au cours des travaux de la Commission, les plus intéressantes furent celles qui avaient trait à ce cadre de référence : par exemple, la pertinence d'introduire une hiérarchie des droits pour que l'égalité entre les hommes et les femmes n'entre jamais en conflit avec la liberté de religion, ou encore la nature de la culture publique commune, au-delà de la langue française et de la charte des droits. Le chapitre central à cet égard est certainement le chapitre VII, "L'intégration dans l'égalité et la réciprocité ": les commissaires, après avoir rappelé l'héritage social et politique de la Révolution tranquille, y proposent une doctrine, l'interculturalisme. Cette approche constitue plutôt un idéal à réaliser qu'un programme social déterminé. Prenant acte de la tension constitutive entre les exigences de l'identité québécoise majoritaire d'origine canadienne-française et la croissance de la diversité ethnoculturelle, ce modèle doit rendre possible une intégration égalitaire et respectueuse de la diversité. Quand ils écrivent que « la crise des accommodements est, en bonne partie, une protestation du groupe ethnoculturel majoritaire soucieux de sa préservation " (p. 119), les commissaires montrent à quelle distance leur analyse se pose d'un modèle purement républicain, au sein duquel n'existerait qu'une seule nation politique et une seule culture, mais ils montrent également leur net refus d'une approche multiculturaliste de l'intégration. L'interculturalisme qu'ils promeuvent représente à la fois une position intermédiaire et une réponse concrète, fondée sur une expérience que le rapport recense longuement, aux défis de l'intégration. Selon les commissaires, le Québec contemporain donne l'exemple d'une identité collective pleinement inclusive et le rapport passe en revue huit voies par lesquelles cette identité évolue actuellement, notamment sur le plan des valeurs communes, la construction d'une mémoire nationale, la formation d'un imaginaire commun dans l'art, la participation civique. Tous ces chantiers sont vivants, actifs et en plein processus de construction et ils soutiennent l'évolution d'une identité collective en mutation. 
Au terme de cette réflexion, les commissaires évoquent cependant le sentiment d'inquiétude maintes fois exprimé devant la Commission au cours de ses travaux. Devant la diversité croissante, ils réaffirment la nécessité d'un pluralisme intégrateur et appellent l'État à en faire la promotion, entre autres par la promulgation d'un texte officiel. Pour le dire autrement, la Commission croit que le temps est venu de donner aux grands textes d'orientation de la Révolution tranquille un complément politique fondamental, sous la forme d'un énoncé qui pourrait garantir les principes de l'égalité et de la réciprocité tout en fournissant aux institutions et aux programmes les balises nécessaires dans le cadre de leur action. De la même manière, le rapport propose un programme de laïcité ouverte, dont les principes devraient être repris dans un livre blanc de la laïcité. Sur ce point, les commissaires endossent entièrement les requêtes de tous les intervenants qui ont souhaité au cours de la Commission que soient précisés les paramètres de la laïcité, notamment au sein des institutions publiques. Comme la suite du rapport le montre, les recommandations mises de l'avant par les commissaires reprennent rigoureusement les mesures découlant de ce vaste cadre de référence et invitent à mettre en ouvre sa philosophie pluraliste. Une analyse détaillée de l'ensemble établirait que la démarche était marquée par un souci de cohérence et un réalisme inspiré d'une écoute attentive du témoignage public.

On ne peut que se réjouir de la parution d'une réflexion aussi ouverte et qui ne se limite pas à prendre en compte les cas, souvent assez banals, qui ont déclenché le travail de la Commission. Mais ce document était à peine public qu'il fut dénoncé comme l'expression d'une position de faiblesse et de compromis face aux revendications qui ont amené la création de la Commission. Le rapport de la Commission Bouchard -Taylor peut-il être relu sans qu'il soit de nouveau, une année s'étant écoulée depuis sa parution, attaqué de toute part comme le porteur de la maladie cosmopolitique, appelée aussi " multiculturalisme ", dont il se démarque pourtant clairement, et comme l'expression d'une trahison du nationalisme traditionnel ? Quand on passe en revue l'ensemble des réactions publiées après le dépôt du rapport au mois de mai 2008 , on est en effet surpris qu'elles soient principalement et quasi exclusivement reliées à l'enjeu identitaire : nous sommes en effet surtout témoins d'une crispation identitaire que les travaux de la Commission n'avaient aucunement pour mandat d'analyser mais qui devint, volens nolens, le pôle inconscient et, selon 
certains, déterminant de la discussion' '. La Commission devait-elle prendre position dans le débat national, promouvoir la souveraineté, écrire la constitution d'un Québec à venir ? Tout cela, et encore plus, ont écrit ceux qui, déçus de lire des analyses complexes sur l'intégration, la laïcité et l'accommodement, n'y ont pas reconnu la prose habituelle du nationalisme. Parmi tous les textes publiés dans la foulée de la parution du rapport, très peu se sont préoccupés du cadre de référence et de la philosophie sociale et politique qui l'inspirait et quasiment aucun n'a pris le soin de revenir sur les pratiques d'harmonisation ou sur les principes de gestion de l'accommodement qui, à défaut d'une discussion des principes, auraient pu retenir l'attention des commentateurs. Presque tous se sont intéressés au contraire au malaise identitaire, à la question du sentiment minoritaire, à l'ethnicisation du rapport social, confirmant en quelque sorte à rebours que les diagnostics posés dans le rapport, loin d'en épuiser la matière, contraignaient d'y revenir.

Relire ce rapport, cela aurait pourtant dû être d'abord faire l'effort d'aller au-delà des susceptibilités de tous ceux, et ils furent nombreux, qui refusèrent de recevoir une proposition d'ouverture et l'énoncé de principes, pour la seule raison que le rapport ne les présentaient pas dans un cadre où la fierté nationale aurait été ravivée par un discours rassurant et " majoritaire ". Etait-ce possible ? On peut en douter quand on voit combien la réception du rapport fut marquée politiquement par la déception, quasi immédiate et unanime, des intellectuels et des politiques souverainistes qui furent prompts à dénoncer son manque de fermeté " nationale ". Là où on aurait attendu un intérêt pour l'analyse des enjeux complexes de l'intégration et une discussion minutieuse des exigences de la laïcité, on n'a pu lire en effet que la réclamation de l'énoncé d'une constitution, ou la réitération d'une épopée de survivance et un hymne aux défricheurs. Bref, le rapport s'avançait sans planter le drapeau attendu, il n'était pas seulement ennuyeux, il ne permettait pas à la " majorité " de se rassurer devant les nouveaux défis du pluralisme. L'ex-premier ministre, M. Bernard Landry, le déclara " déplorable " deux jours après la publication, la chose était entendue.

$$
+4
$$

1. Un des premiers textes publiés à la parution dénonce cette "surenchère identitaire " au sein des partis politiques qui ont cherché à tirer avantage de l'inquiétude face aux accommodements, notamment l'ADQ; il s'agit du texte collectif du comité de rédaction de la revue Les cahiers du 27 juin, "Rapport de la Commission Bouchard-Taylor. L'irresponsabilité politique et la surenchère identitaire ", Le Devoir, 26 mai 2008, http://www.ledevoir.com/2008/05/26/191312.html (3 avril 2009). Je m'accorde avec la position générale de cette déclaration, qui déplore l'abstention politique durant le débat. 
On peut regretter que les commissaires n'aient pas prévu cette réaction et introduit les nuances pour s'en prémunir. Cela aurait été aisé. Mais on peut aussi penser au contraire qu'ils ont eu le courage de ne pas flatter une opinion nationale dont ils ont eu, deux fois plutôt qu'une, l'occasion d'entendre les doléances. C'est la raison pour laquelle, pour ne citer qu'un seul article, le reproche du sociologue Guy Rocher ${ }^{2}$ peut être posé comme le symptôme le plus sensible de l'impossibilité de lire ce rapport au moment de sa réception. Quel est ce symptôme ? Reconnaissant la générosité de l'intention qui anime le rapport et propose de faire du Québec une terre d'accueil où le racisme sera combattu et les inégalités réduites, Guy Rocher ne considère cependant rien d'autre que le sort infligé à la fierté nationale : s'attardant au seul fait que le rapport évoque plusieurs fois l'inquiétude d'une population " minoritaire ", il déplore que la population ne soit pas décrite comme une société majoritaire. Ciblant des expressions telles que "l'inquiétude du minoritaire ", le sociologue omet sciemment de noter que les commissaires, dès l'ouverture de leur rapport, ont replacé la nation québécoise comme minorité de langue française au Canada et en Amérique du Nord. S'ils reprennent souvent l'expression de cette inquiétude dans le rapport, c'est pour donner tous ses droits à la revendication identitaire du Québec au sein même de ce vaste ensemble multiculturel que constituent le Canada et l'Amérique du Nord, et non pour l'y enfoncer au point de l'anéantir, et encore moins pour le soumettre aux exigences de la diversité. On voit donc, par ce banal exemple, à quel point une lecture du rapport dans ses dimensions d'ouverture était, quasi a priori, impossible pour ceux qui en attendaient d'abord et avant tout un manifeste de l'identité dite majoritaire et un argumentaire de résistance à l'inflation des demandes des communautés. Nous sommes ici au confluent de préoccupations dont on peut se demander, constatant la gravité du préjugé et du refus de lire, si elles pouvaient conduire à autre chose qu'à une profonde incompréhension mutuelle. Jacques Beauchemin, qui faisait partie comme moi du comité conseil de la Commission, et qui ne peut être soupçonné d'en méconnaître la réflexion, déclara une semaine avant la parution, que les commissaires erraient en exigeant de la population qu'elle se conforme aux vertus du multiculturalisme ${ }^{3}$.

$$
+4+
$$

2. Guy ROCHER, "Rapport Bouchard-Taylor. Une majorité trop minoritaire ? ", Le Devoir, 12 juin 2008 , http://www.ledevoir.com/2008/06/12/193612.html (3 avril 2009).

3. Dans une interview au journaliste Jeff $\mathrm{HEINRICH}$, lequel eut copie de larges morceaux du rapport avant sa parution : "Dissident decries "whitewash" ", The Gazette, 17 mai 2008, http://www2.canada.com/ montrealgazette/news/story.html?id=d86da0a9-822d-454e-be4e-a87cdee372f9 (3 avril 2009). 
Le commissaire Gérard Bouchard dut en faire le constat désabusé dans une lettre ouverte où il exprima sa déception ${ }^{4}$.

L'inquiétude dont il est question, autant dans le rapport que dans les réactions comme celle de Guy Rocher, doit être prise au sérieux. Si on propose en effet, comme le suggère Guy Rocher, de la considérer comme l'inquiétude du majoritaire qui prend conscience de son nouveau "statut de majoritaire ", en accédant dans la modernité à une forme de maturité politique découlant de la Révolution tranquille, c'est parce qu'on croit possible de penser cette inquiétude autrement. Par exemple, comme l'expression des difficultés d'ajustement au nouveau contexte identitaire de la mondialisation. Cette manière de présenter l'enjeu me semble très paradoxale : les majorités sont en général peu inquiètes de leur statur, elles en tirent plutôt une légitimité accrue et plus de pouvoir. Comment expliquer que cette inquiétude ait $\mathrm{pu}$, dans un contexte majoritaire, engendrer la crise des accommodements raisonnables, sinon par la perception d'une menace, par l'expérience d'une anxiété, et dans certains cas par une réelle détresse face à l'immigration et au pluralisme croissant de la société ? Ces réflexes, exprimés trop souvent pour qu'on les pousse à la marge dans le témoignage entendu à la Commission, sont ceux d'une population qui se perçoit comme une minorité menacée dans un ensemble dont elle ne maîtrise pas l'évolution et ne contrôle plus les normes. Les commissaires ont analysé ce témoignage et ils ont eu raison de le resituer dans un cadre plus vaste, qui seul l'explique : alors que les combats de la Révolution tranquille ont conduit à un Etat solide et en apparence sûr de lui, la peur de la disparition refait surface quand cette identité reconquise se trouve de nouveau agressée par les exigences, perçues comme démesurées ou illégitimes, des minorités ethnoculturelles. Les sociologues et les historiens de la nation auront beau réaffirmer le succès de la Révolution tranquille et, comme Guy Rocher, parler d'une prise de conscience du statut majoritaire, cette inquiétude demeure et c'est elle qui se trouve à la racine de cette crise. Pourquoi refuser de la voir et surtout de la nommer ?

Contrairement à ce qu'affirme Guy Rocher, je ne trouve personnellement aucune ambiguïté dans le rapport de la Commission; c'est plutôt de complexité qu'il faudrait parler, et de la nécessité de faire intervenir dans l'analyse des accommodements l'ensemble des facteurs qui agissent

$++$

4. Gérard BOUCHARD, "Gérard Bouchard réplique à ses détracteurs. Le débat prend une tournure inquiétante ", Le Devoir, 10 juin 2008, http://www.ledevoir.com/2008/06/10/193341.html (3 avril 2009). 
aujourd'hui sur l'évolution des normes du vivre ensemble, et pas seulement la réaffirmation des acquis de la majorité ou les promesses de la modernité. J'irais cependant plus loin, en exprimant à mon tour une réserve d'une autre nature sur le recours au lexique de la majorité et de la minorité, qui semble pour les intellectuels nationalistes porteur de l'évidence sociologique de l'époque à condition qu'il soit utilisé de manière favorable. Il est certes indispensable, dans le contexte politique du souverainisme nationaliste, de maintenir ce lexique, autant pour sa valeur explicative dans l'analyse des rapports sociaux que pour sa fonction dans la rhétorique identitaire. C'est la raison pour laquelle le fait que le rapport ait évoqué la notion de "québécois d'origine canadienne-française " a choqué tant de lecteurs du rapport, précisément dans les milieux où cette étiquette est pourtant nécessaire dans l'analyse historique 5 . Ce n'est pas en effet en provenance des milieux qui eux sont véritablement minoritaires, comme les communautés culturelles issues de l'immigration, voire la population anglophone, que la critique de cette caractérisation est venue : non, elle est venue des intellectuels nationalistes eux-mêmes. On se trouve ici en face d'une contradiction performative qui ne peut qu'intéresser les lecteurs du rapport. Faisons l'hypothèse que le rapport aurait fait l'économie de toute dimension ethnoculturelle, et fait fond sur une identité purement citoyenne libérée de toute attache ethnicisante, quel sens y aurait-il eu à faire état des demandes d'accommodement et à parler de la majorité ? C'est donc à l'ensemble de cette conceptualité que nous sommes invités à renoncer au nom d'un idéal citoyen incontestable ${ }^{6}$. Mais cet idéal, qui fonctionne ici comme une utopie républicaine, ne peut servir à décrire les groupes en présence. Un tel renoncement est non seulement une impossibilité pragmatique, il n'est pas souhaité par ceux-là mêmes qui s'en font les promoteurs.

Nous retrouvons ici la même structure paradoxale que celle que j'ai tenté d'identifier au sujet de la minorité : tendez à ceux qui revendiquent cette identité le miroir où ils pourront en reconnaître le discours, ils seront les premiers à le refuser. Pourquoi ? Ce nouveau paradoxe vient peut-être éclairer le premier : il nous montre en effet que si, aux yeux de ses critiques, le rapport a eu tort de recourir au lexique de la majorité et des minorités

$$
+4
$$

5. L'historien Jocelyn LÉTOURNEAU, comme souvent par ailleurs, fait ici exception. Voir son appui au rapport : "La raison de Bouchard et Taylor ", Le Devoir, 19 juin 2008, http://www.ledevoir.com/ 2008/06/19/194616.html (3 avril 2009).

6. Voir en ce sens les remarques de Gilles BOURQUE, "Bouchard-Taylor: un Québec ethnique et inquiet ", Le Devoir, 30 juillet 2008, http://www.ledevoir.com/2008/07/30/199693.html (3 avril 2009). 
pour décrire une appréhension qui est pourtant l'expérience la plus ordinaire et la plus courante de l'inquiétude recueillie lors des travaux de la Commission et qui ne peut être saisie autrement, il a eu encore plus tort d'associer cette inquiérude du minoritaire à l'histoire nationale, et en particulier au fait que cette histoire de la majorité soit celle des Québécois d'origine canadienne-française. Relisons à cet égard l'analyse de Guy Rocher, qui révèle sur ce point ses présupposés et ses attentes. Si cette majorité avait pu témoigner de toute l'assurance dont on souhaite la créditer, où aurait résidé le problème ? Si elle avait en main le dispositif politique et la maturité pour intégrer les demandes des communautés et si elle avait confiance dans le destin de la langue française dans une société minorisée de manière évidente sur ce plan en Amérique du Nord, pourquoi s'est-elle engagée dans cette longue conversation nationale avec autant d'humilité que d'insécurité ? Tout le paradoxe de la critique est là : reprocher au constat posé par les commissaires sur cette ambivalence de l'avoir nommée et d'en avoir tenu compte, au lieu de la nier dans une sorte de volontarisme et de pédagogisme dont nous sommes par ailleurs coutumiers.

L'analyse de Guy Rocher est à la fois lucide et fragile. Lucide, elle l'est d'abord dans la reconnaissance des nouveaux équilibres sociaux en voie de formation dans une société où d'une part la jeunesse ne connaît plus, ou pas de la même manière, les sentiments d'oppression du minoritaire qui caractérisaient la génération d'avant la Révolution tranquille et où, d'autre part, les " minorités ethnoculturelles " s'adressent, confiantes, à la société majoritaire pour lui demander des accommodements en matière de culture et de religion autant que l'énoncé de principes et de balises clairs pour l'avenir. Si je parle ici de lucidité, c'est parce qu'en peu de mots Guy Rocher décrit les deux grands volets du récit entendu, jour après jour, à la Commission. La jeunesse est confiante, assurée de son identité et forte de ses racines, et finalement assez peu concernée par la gestion d'un pluralisme qui lui paraît à la fois naturel et nécessaire. Les solutions lui paraissent à portée de main, l'intégration est une responsabilité dont elle a confiance de pouvoir s'acquitter. Ce n'est pas dans cette jeunesse en effet qu'on entendra la récrimination du " minoritaire ", bien au contraire, comme l'ont démontré les interventions des jeunes devant la Commission.

Il n'est pas moins vrai que, devant l'expression de cette anxiété dans la portion dite "majoritaire " de la population, plusieurs représentants de communautés culturelles ont renforcé leurs revendications, dans divers secteurs ouverts à l'accommodement et développé une expertise notable de chartiste. Parmi eux, des groupes ont fait valoir des demandes interprétées 
comme exorbitantes par cette majorité, notamment toutes ces demandes perçues comme remettant en cause l'égalité des sexes et qui ont conduit des critiques à exprimer la crainte que les principes de la charte soient en péril ${ }^{7}$. Les acquis de la Révolution tranquille à cet égard seraient-ils si fragiles que la seule présence d'un foulard sur la tête d'une enseignante dans une école pourrait menacer la laïcité ? Certains l'ont soutenu. Mais si cette analyse perçoit avec justesse les forces en présence, pourquoi est-elle si fragile? Parce qu'elle est liée à une attente politique relative aux minorités culturelles : selon Guy Rocher, les commissaires auraient mieux fait de rassurer la majorité, de manière à faciliter l'intégration et l'interculturalisme dont ils ont fait le maître-mot de leur rapport. Ce faisant, ils auraient été meilleurs pédagogues. En exposant honnêtement et rigoureusement l'anxiété dont ils ont recueilli le témoignage, ils ont conforté une représentation qui ne pouvait que soutenir la demande des minorités.

Trahirai-je la position de Guy Rocher et de ceux qui l'ont suivi en disant qu'ils auraient préféré que cette anxiété soit passée sous le tapis et que le rapport flatte la majorité historique ? A mes yeux, cette position n'aurait été ni juste ni fidèle aux travaux de la Commission. Je crois au contraire que le rapport a rempli les deux volets de son mandat en toute rigueur : d'une part, les commissaires ont resitué dans un cadre susceptible d'en relativiser l'importance l'ensemble des affaires relatives aux accommodements. La moitié du rapport est consacrée à une analyse minutieuse de ces pratiques et aux principes qui permettent de les encadrer : cette partie du rapport, à sa relecture, demeure très fructueuse, et elle aura pour effet de stimuler la réflexion concrète sur ces situations, dans toutes les institutions publiques, et notamment dans l'école, où les demandes d'accommodement sont complexes et urgentes. Mais les commissaires se sont aussi acquittés exemplairement de cette partie de leur tâche qui touche à la réflexion sur le cadre de référence de la vie collective. Quand on relit les chapitres centraux sur l'intégration et la laïcité, on y trouve une réflexion de grande importance et une philosophie politique qui n'a rien à voir avec les portraits déformés qu'en ont fournis les journaux après la parution du rapport. Je doute que même un ancien premier ministre ait eu le temps de lire ces textes complexes en deux jours. Il y faut du temps et même si un nombre infime des recommandations

$$
++*
$$

7. C'est le cas de Yolande GEADAH, dans son essai Accommodements raisonnables. Droit a la difference et non difference des droits, Montréal, VLB éditeur, 2007. Voir également son intervention, à mon avis un peu alarmiste: "Un rapport insensible à l'égalité des sexes", Le Devoir, 16 juin 2008, http:// www.ledevoir.com/2008/06/16/194176.html (3 avril 2009). 
du rapport semble avoir trouvé l'oreille du Législateur, ce n'est pas une raison pour considérer comme inutile ou inopérant l'ensemble de ce rapport. J'ai eu le privilège de participer aux travaux de la Commission, j'y ai trouvé un laboratoire de philosophie sociale et politique d'une exceptionnelle fécondité et je pense que, une année plus tard, il est temps de retourner aux analyses de ce rapport et d'en discuter soigneusement les conclusions.

Georges Leroux

Université du Québec à Montréal

\section{Bernard Andrès et Gérard Bouchard (dir.) Mythes et sociétés des Amériques \\ Montréal, Québec Amérique, 2007.}

L'intérêt que nous portons, au Québec, à l'Amérique (et en particulier aux Etats-Unis) n'est pas neuf. En se limitant au plan culturel, selon une définition assez stricte du terme, il est certainement possible de remonter au moins jusqu'à l'Institut canadien. La réflexion sur les Amériques, sur ce qu'on nomme maintenant tout naturellement "l'américanité ", est évidemment beaucoup plus récente, datant d'à peine quelques décennies. En gardant en tête le fait que les dates précises, lorsqu'il s'agit de parler du développement d'un concept, ont toujours quelque chose d'un peu aléatoire, on peut se demander néanmoins si la défaite du " oui » en mai 1980 n'a pas un rôle symbolique à jouer dans ce renouvellement d'intérêt pour les Amériques, tant il est vrai que le référendum était associé au "fait français " et, par métonymie, à la France elle-même - par conséquent à l'Europe. Se tourner vers les "terres intérieures" (Pierre Nepveu a intitulé un de ses essais Intérieurs du Nouveau Monde) plutôt que de l'autre côté de l'Atlantique aurait été une façon d'oublier ce qui avait été ressenti comme une défaite par une nette majorité d'écrivains et d'intellectuels francophones. Sans aller trop loin dans cette intuition, il faut rappeler que les romans "américains" publiés par des francophones québécois dans les années subséquentes se sont multipliés. On peut même dire que Jacques Poulin, auteur jusque-là relativement peu connu, a été vraiment consacré après la parution en 1984 de Volkswagen Blues, road book se déroulant de Gaspé à San Francisco, devenu un peu le roman phare de la décennie.

Une trentaine d'années après ce nouveau souffle donné aux analyses de nos relations intellectuelles avec les Amériques, Mythes et societés 